\title{
Survival Analysis of Twin Under Five Mortality in Ethiopia: Using Gamma Frailty Modelling Approach
}

\author{
Mesfin Haileyesus* \\ Department of Statistics, College of Natural and Computational Sciences, \\ Dire Dawa University, P.O. Box 1362, Ethiopia \\ Dereje Meretu \\ Department of Statistics, College of Natural and Computational Sciences, \\ Dire Dawa University, P.O. Box 1362, Ethiopia
}

\begin{abstract}
Twins are relatively rare events, but several studies confirm that they contribute substantially to mortality in both neonatal and post-neonatal periods. The excess infant and child mortality rates among twins calls for a need to identify the main causes behind it. In studies involving multiple individuals from the same family, the independence assumption is not plausible unless all important familial factors were measured and controlled for in the model. Hence, it is important to include random effect or frailty in the model to account for the existence of correlation or unmeasured covariates. This study, therefore, employed survival analysis using gamma frailty models. Information on twin under-five mortality was found from the birth history of women who were included in the 2016 Ethiopia Demographic and Health survey. The results of the study show that sex of child, between twins birth order, preceding birth interval, and succeeding birth interval are significantly associated with underfive twin mortality. In addition, the result of the study confirmed the significance of shared /family random effect. Keywords: word; under-five twin mortality; frailty; random effect; gamma
\end{abstract}

DOI: $10.7176 / \mathrm{JBAH} / 9-15-05$

Publication date: August $31^{\text {st }} 2019$

\section{Introduction}

The rate of occurrence of infant and child mortality rates reflects the country's level of socio economic development and quality of life. According to the WHO's report, 5.9 million Children under age five died in 2015 and in average 16000 children under age five dies every day. The hazard of death for child under-five years is still highest in African Region (81 per 1000 live births), this is approximately 7.37 times the risk in European Region (11 per 1000 live births). Fortunately, under-five mortality rate decreased by 53\%, from an estimated rate of 91 deaths per 1000 live births in 1990 to 43 deaths per 1000 live births in 2015 (WHO, 2016).

There are huge differences in child mortality among low and middle income countries and the industrial world with Sub- Saharan Africa and South East Asia carrying the highest burden of under-five mortality (You, New, \& Wardlaw, 2014; WHO, 2006). The highest rates of child mortality are still in Sub-Saharan Africa-where 1 in 8 children dies before age 5, more than 17 times the average for developed regions ( 1 in 143)-and Southern Asia (1 in 15) (UNICEF, 2011). Ethiopia being one these country under five mortality still remain high, 88/1000 live birth (CSA, 2012) .

Sub-Saharan Africa not only has the highest rate of twin births in the world (Smits \& Monden, 2011) but also the world's highest rate of infant and child mortality (WHO, 2016). Twin births contribute substantially to infant and child mortality in both neonatal and post-neonatal periods (Alam, Van Ginneken, \& Bosch, 2007).

In Sub-Saharan African countries, there are few studies that evaluate the mortality of twins after the neonatal period. All of these studies showed the excess mortality of twin compared to singleton (Guo \& Grummer-Strawn, 1993; Pison, 1992; Justesen \& Kunst, 2000; Olalekan, Mubashir, \& Ismail, 2000). Close contact between twin babies increases the chance of cross-infections (Justesen \& Kunst, 2000). In addition, worldwide there are several studies on infant and child mortality showed that infant and child mortality is mainly associated twin birth (Koissi \& Hgns, 2001; Munyamahoro, 2017; Bereka, Habtewold, \& Nebi, 2017) among others. Furthermore, in studies involving multiple individuals from the same family (e.g. twins), the presence of correlation is expected, and obviously independence assumption is not plausible unless all important familial factors were measured and controlled for in the model. This is because, children belong to the same family share certain unobserved characteristics (or heterogeneity), which may not be sufficiently described by the covariates included in the models. The ignorance of such family-level correlation may lead to biased parameters estimates (Guo \& Rodriguez, 1992).

The persistence of high level of infant and child mortality rates among twins call for a need to identify the determinants of twins mortality separately. Identifying all significant determinants of twin birth child mortality is essential to form policies and strategies to accelerate the reduction of child mortality and also to meet the United Nations the sustainable development goal (SDGs) (i.e., aiming to reduce under-five mortality to at least as low as 
25 per 1000 live births by 2030). This study, therefore, aims to identify the determinants of under-five twin mortality in Ethiopia using frailty modelling approach that can take the presence of the unobserved family random effect into account.

\section{Materials and Methods}

\subsection{Data and data source}

This study used the 2016 Ethiopia Demographic and Health survey. The 2016 Ethiopia Demographic and Health Survey (EDHS) is the fourth Demographic and Health Survey conducted in Ethiopia. It was implemented by the Central Statistical Agency (CSA) at the request of the Federal Ministry of Health (FMoH). Data collection took place from January 18, 2016, to June 27, 2016. The standard DHS survey consists of a household questionnaire and a women's questionnaire administered to a nationally representative sample of women aged 15-49 years. The women's questionnaire is used to gather information on complete birth histories to estimate infant and child mortality probabilities. Information on twin mortality was found from the birth history of women who were included in the survey. All twin births before 2011 were included.

\subsection{Study design}

The study design was a retrospective study design.

\subsection{Variables of the study}

The response or outcome variable for this study is the survival time of a pair of under-five twins measured in months. Among the various covariates that are thought to have effect on survival of a twin child, those time invariant factors such as mothers age at child birth (below18 years, between 18-35 years and above 35 years), sex of the child, preceding birth interval ( $1^{\text {st }}$ born ,below 18 months, between 18-24 months and above 24 months), succeeding birth interval (below 18 months, between 18-24 months, above 24 months and last born), between twins birth order (first born, second born) and less likely to be time variant factor such as residence (urban, rural) were included in this study.

\subsection{Method of Data analysis}

Descriptive statistics tools such as frequency, percentage and cross tabulation was used to present the data. The log rank test was performed to test if there are statistically significant differences among the survival experience of the different groups of the covariates. When proceed to model fitting, first, the usual Cox Proportional Hazard (PH) model was fit including all the potential risk factors that were significant in log rank test. Then, univariate and shared gamma frailty variable (random effect) was introduced on the full multivariable Cox PH model to assess the presences and significances of individual and shared frailty. Likelihood ratio test was employed to test the significance of the random effect. The level of significance for all the inferential statistics was set at $p<0.05$. Statistical R software was used.

\section{Results}

A total of 908 or (454 pairs of) twin child deliveries were recorded in the 2016 Ethiopia Demographic and Health survey. The data on 622 children or (311 pairs of twin) born before 2011 were analyzed in this study. The overall information on censoring and covariates that are included in this study are presented below. 
Table 1.Descriptive summaries of variables of the study

\begin{tabular}{|c|c|c|c|}
\hline & & \multicolumn{2}{|c|}{ Child status } \\
\hline & & Alive & Dead \\
\hline Covariates & Frequency $(\%)$ & Frequency $(\%)$ & Frequency $(\%)$ \\
\hline \multicolumn{4}{|l|}{ Age of mother at child birth } \\
\hline$<18$ years & $42(6.8)$ & $13(31.0)$ & $29(69.0)$ \\
\hline $18-35$ years & $542(87.1)$ & $313(57.7)$ & $229(42.3)$ \\
\hline$>35$ years & $38(6.1)$ & $15(39.5)$ & $23(60.5)$ \\
\hline \multicolumn{4}{|l|}{ Residence } \\
\hline Urban & $104(16.7)$ & $58(55.8)$ & $46(44.2)$ \\
\hline Rural & $518(83.3)$ & $283(54.6)$ & $235(45.4)$ \\
\hline \multicolumn{4}{|l|}{ Sex } \\
\hline Male & $345(55.5)$ & $178(51.6)$ & $167(48.4)$ \\
\hline Female & $277(44.5)$ & $163(58.8)$ & $114(41.2)$ \\
\hline \multicolumn{4}{|l|}{ Between twins' birth order } \\
\hline First born & $311(50.0)$ & $190(61.1)$ & $121(38.9)$ \\
\hline Second born & $311(50.0)$ & $151(48.6)$ & $160(51.4)$ \\
\hline \multicolumn{4}{|l|}{ Preceding birth interval } \\
\hline No precede sibling & $116(18.7)$ & $60(51.7)$ & $56(48.3)$ \\
\hline$<18$ months & $74(11.9)$ & $19(25.7)$ & $55(74.3)$ \\
\hline 18-24 months & $117(18.8)$ & $67(57.3)$ & $50(42.7)$ \\
\hline$>24$ months & $315(50.6)$ & $195(61.9)$ & $120(38.1)$ \\
\hline \multicolumn{4}{|l|}{ Succeeding birth interval } \\
\hline Last born or no child after & $94(15.1)$ & $63(67.0)$ & $31(33.0)$ \\
\hline$<18$ months & $105(16.9)$ & $31(29.5)$ & $74(70.5)$ \\
\hline 18-24 months & $110(17.7)$ & $63(57.3)$ & $47(42.7)$ \\
\hline$>24$ months & $313(50.3)$ & $184(58.8)$ & $129(41.2)$ \\
\hline
\end{tabular}

As can be seen from Table 1, regarding age of mother at child birth, in the first category below 18 years, there were $6.8 \%$ of the study population, (of whom $69 \%$ of the children have died and $31 \%$ are alive); $6.1 \%$ of the children were born from mothers whose age exceeds 35 years, (of whom $60.5 \%$ of the children have died and $39.5 \%$ are alive); and the remaining $87.1 \%$ children belong to mothers whose age at birth was between $18-35$ years (of whom $42.3 \%$ of have died and $57.7 \%$ are alive). Regarding place of residence, $16.7 \%$ were from urban area (of which $55.8 \%$ are alive and $44.2 \%$ are dead); the rest $83.3 \%$ were from rural area (of which $54.6 \%$ are alive and $45.4 \%$ are dead). Regarding the sex of children, $55.5 \%$ were male (of which $51.6 \%$ are alive and $48.4 \%$ are dead); the rest $44.5 \%$ were female (of which $58.8 \%$ are alive and $41.2 \%$ are dead). Among the first born twin children, $61.1 \%$ of them are alive and $38.9 \%$ are dead; while from the second born twin children, $51.4 \%$ are dead and $48.6 \%$ are alive.

Regarding preceding child's birth order, $18.7 \%$ of the children were first born (of which $51.7 \%$ are alive and $48.3 \%$ are dead); $11.9 \%$ were born before their older sibling reaches the age 18 months (of which $25.7 \%$ are alive and $74.3 \%$ are dead) ; $18.8 \%$ of the children were born when their older sibling's age was between $18-24$ months (of which $57.3 \%$ are alive and $42.7 \%$ are dead); and the remaining $50.6 \%$ of the study population have older sibling whose age exceed 24 months (of which $61.9 \%$ of the children are alive and $38.1 \%$ are dead).

Regarding the succeeding birth interval, $15.1 \%$ were last born or no child after (of which $67 \%$ are alive and $33 \%$ are dead); $16.9 \%$ were having a succeeding birth interval below 18 months (of which $29.5 \%$ are alive and $70.5 \%$ are dead); $17.7 \%$ were having a succeeding birth interval between 18 months and 24 months (of which $57.3 \%$ are alive and $42.7 \%$ are dead); and the rest, $50.3 \%$ were having a succeeding birth interval of more than 24 months (of which $58.8 \%$ are alive and $41.2 \%$ are dead).

Table 2.Log-Rank Test for covariates

\begin{tabular}{llll}
\hline Independent variables & Test statistic & Df & p-value \\
\hline Age of mother at birth & 20.1 & 2 & $<0.001^{*}$ \\
Residence & 0.1 & 1 & 0.731 \\
Sex & 5.2 & 1 & $0.022^{*}$ \\
Between twins' birth order & 9.1 & 1 & $0.002^{*}$ \\
Preceding birth interval & 38.2 & 3 & $<0.001^{*}$ \\
Succeeding birth interval & 39 & 3 & $<0.001^{*}$ \\
\hline
\end{tabular}

To test if there are statistically significant differences among the survival experience of the different groups of the covariates, the log rank test is performed. As one can see from Table 2, the results of the log rank test indicate that there are significant differences between the survival experiences of different groups for all the 
covariates except residence.

The log rank test was also used as a univariate analysis to check the significance of incorporating each one of the independent variables in a model that contains several (multiple) independent variables. If a given independent variable is insignificant in log rank test, then it is unnecessary to incorporate the variable in model fitting that contains multiple independent variables such as Cox PH model.

The Cox PH model that employed in several child mortality studies assume a homogeneous population. In other words, Cox PH assume that all individuals sampled into that study are subject in principle under the same risk. Although Cox PH model's independence assumption is not plausible, for making comparison we fit Cox PH model. All independent variables that were found to be significant in log rank test were included in the model. The results are given in Table 3

Table 3.Results of the Cox PH model

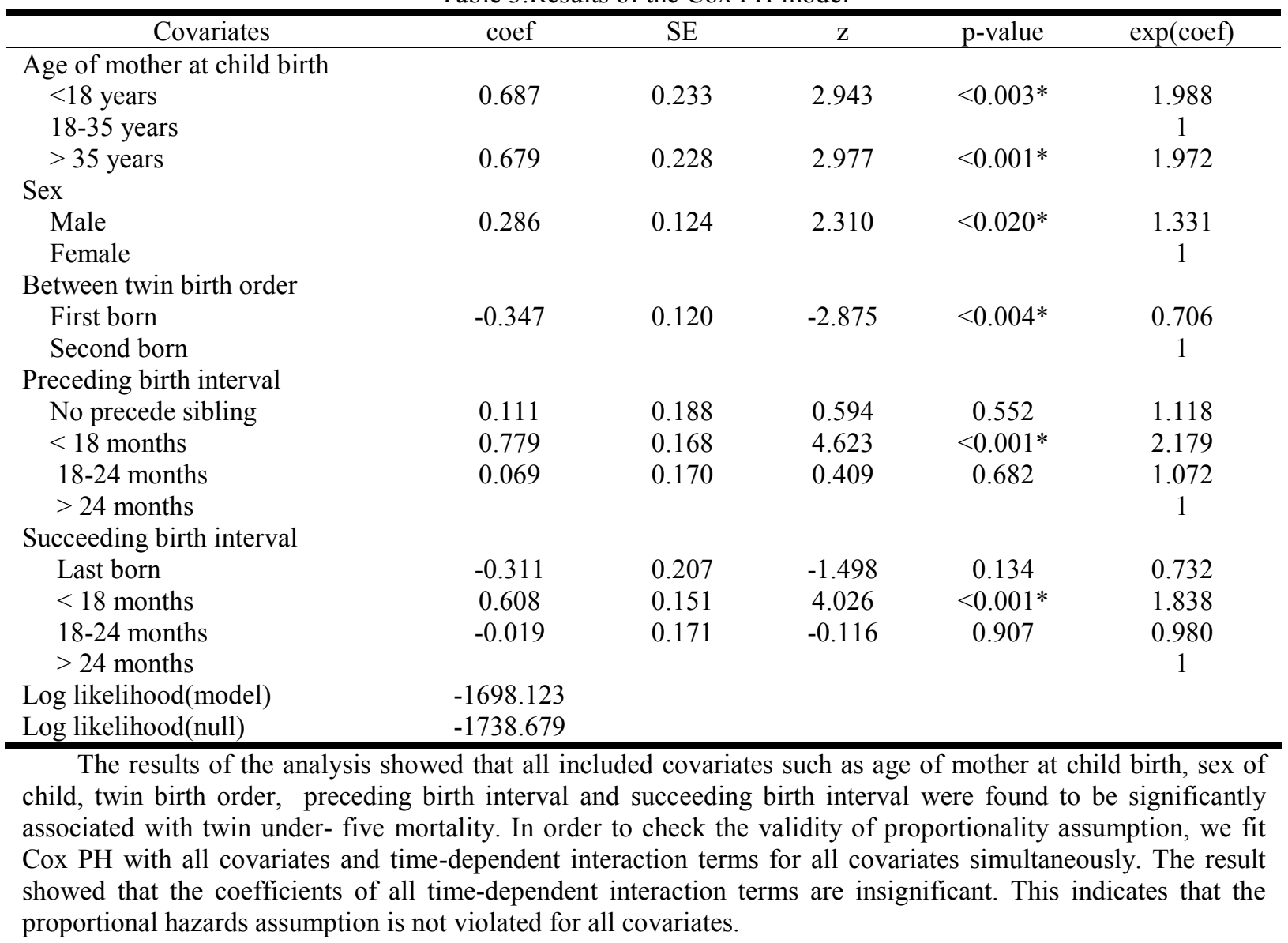


Table 4.Results of the shared gamma frailty model

\begin{tabular}{|c|c|c|c|c|c|c|}
\hline Covariates & coef & $\mathrm{SE}$ & Chisq & p-value & $\exp (\operatorname{coef})$ & $95 \%$ CI $\exp ($ coef $)$ \\
\hline \multicolumn{7}{|c|}{ Age of mother at child birth } \\
\hline$<18$ years & 0.689 & 0.454 & 2.30 & 0.129 & 1.993 & $0.818-4.858$ \\
\hline $18-35$ years & & & & & 1 & \\
\hline$>35$ years & 0.809 & 0.467 & 2.99 & 0.083 & 2.246 & $0.897-5.620$ \\
\hline \multicolumn{7}{|l|}{ Sex } \\
\hline Male & 0.550 & 0.179 & 9.45 & $0.002 *$ & 1.733 & $1.220-2.462$ \\
\hline Female & & & & & 1 & \\
\hline \multicolumn{7}{|l|}{ Between twin birth order } \\
\hline First born & -0.438 & 0.126 & 12.01 & $<0.001 *$ & 0.644 & $0.503-0.826$ \\
\hline Second born & & & & & 1 & \\
\hline \multicolumn{7}{|l|}{ Preceding birth interval } \\
\hline No precede sibling & 0.173 & 0.331 & 0.27 & 0.601 & 1.189 & $0.621-2.275$ \\
\hline$<18$ months & 0.946 & 0.336 & 7.92 & $0.004 *$ & 2.575 & $1.332-4.977$ \\
\hline 18-24 months & 0.148 & 0.304 & 0.24 & 0.627 & 1.159 & $0.637-2.107$ \\
\hline$>24$ months & & & & & 1 & \\
\hline \multicolumn{7}{|l|}{ Succeeding birth interval } \\
\hline Last born & -0.484 & 0.362 & 1.79 & 0.180 & 0.615 & $0.303-1.252$ \\
\hline$<18$ months & 0.618 & 0.288 & 4.60 & $0.031 *$ & 1.856 & $1.054-3.266$ \\
\hline 18-24 months & -0.135 & 0.309 & 0.19 & 0.660 & 0.872 & $0.476-1.600$ \\
\hline$>24$ months & & & & & 1 & \\
\hline$\sigma_{z}^{2}$ & 1.969 & & & & $<0.001$ & \\
\hline Log likelihood(model) & -1437.435 & & & & & \\
\hline Log likelihood(null) & -1738.679 & & & & & \\
\hline
\end{tabular}

The Cox's regression model cannot take the presence of the unobserved heterogeneity in the population into account. As a result parameter estimates are often biased when some influential factors are not observed .To account for unobserved heterogeneity this model should be modified to include hidden frailty together with observed covariate (Iachine, 1995). Each child has a proper susceptibility to infection, independently of his family membership. In addition, inside the common global family behavioural factor, parents may adopt a slightly different prenatal and neonatal attitude from one child to the next (Childs, Moxon, \& Winkelstein, 1992).

Thus, univariate semi-parametric gamma frailty model was fit to assess the presence of unobserved heterogeneity at individual (child) level. The results of the univariate gamma frailty model were found to be exactly similar to the results of the Cox PH model given in Table 3 . This is because the estimate of the heterogeneity parameter $\sigma_{z}^{2}$ became 5e-07 that is almost zero. And the results of the hypothesis test about the significance of the variance of the random effect i.e. $H_{0}: \sigma_{z}^{2}=0$ versus $H_{1}: \sigma_{z}^{2}>0$ found to be insignificant. This indicates that, there is no significant unobserved heterogeneity in the study population (at individual level). As a result, the estimates of covariate effect are exactly the same to the estimates of the Cox PH model given Table 3

Then shared gamma frailty is introduced on the full multivariable Cox PH model to assess the random effect shared by the twins. The result is given in Table 4

Comparison of the two models was made to decide which model fit the data better. As can be seen from Table 5, the shared gamma frailty model has the highest log likelihood and minimum AIC and BIC values, indicating that this model fits the data better than the Cox PH model which did not take in to account the shared random effect. Furthermore, as can be seen from Table 4 an estimate of the heterogeneity parameter $\sigma_{z}^{2}$ is 1.969 . Since Cox PH model is nested within shared gamma frailty model we conduct the likelihood ratio test for the significance of the heterogeneity parameter i.e. $I I_{0}: \sigma_{z}^{2}=0$ versus $I I_{1}: \sigma_{z}^{2}>0$. As can be seen from Table $4, H_{0}: \sigma_{z}^{2}=0$ is rejected with $\mathrm{p}$ value $<0.001$ indicates that one cannot ignore the correlation between the pair of twins. Hence, shared gamma frailty model fit the data better than the Cox PH model which did not take in to account the presence of correlation.

Table 5.Comparison of Cox PH and Shared frailty model

\begin{tabular}{|l|l|l|l|l|}
\hline Model & Log-likelihood (null) & Log-likelihood (model) & AIC & BIC \\
\hline Cox PH & -1738.679 & -1698.123 & 3416.245 & 3452.629 \\
\hline Shared gamma frailty & -1738.679 & -1437.435 & 2876.321 & 2878.963 \\
\hline
\end{tabular}

The results of the analysis using shared gamma frailty model given in Table 4 showed that covariates such as sex of child, twin birth order, preceding birth interval and succeeding birth interval were found to be significantly associated with twin under- five mortality. However, the incorporation of a shared gamma frailty 
variable make the variable age of mother at child birth insignificant at $5 \%$ level of significance.

As can be seen from Table 4, the estimated hazard ratio of a male twin child (i.e. a child of twin birth) is 1.733 (95\% CI: 1.220 -2.462) implying that the risk of dying for a male twin child is $73.3 \%$ higher than a female twin child controlling for the other covariates in the model. This figure can be as low as $22.0 \%$ and as high as $146 \%$ with $95 \%$ confidence.

The estimated hazard ratio of a first born twin child is 0.644 (95\% CI: 0.503-0.826) implying that the risk of dying for a first born twin child is $35.6 \%$ less likely than a second born twin child (reference group) controlling for the other covariates in the model.

The estimated hazard ratio a child of twin birth who was born before the older sibling reaches the age of 18 months is 2.575 (95\% CI: 1.332-4.977). Thus, the hazard rate of a child of twin birth who was born before the older sibling reaches the age of 18 months is 2.575 times than a child of twin birth who born when the older sibling's age exceeds 24 months (reference group) controlling for other covariates in the model. The confidence interval indicates that the risk of dying can be as low as 1.332 times and as high as 4.977 times than that of the reference group.

The estimated hazard ratio a child of twin birth who has a younger sibling that born within 18 months is 1.856 (95\% CI: 1.054-3.266). This implies that the risk of dying for a child of twin birth who has a younger sibling that born within 18 months is $85.6 \%$ higher than a child of twin birth who has a younger sibling that born after 24 months controlling for the other covariates in the model. This figure can be as low as $5.4 \%$ and as high as $226 \%$ with $95 \%$ confidence.

\section{Discussion}

The main aim of this study was obtaining the estimates of covariate effect on survival of under-5 twins. Since this study was about survival of under-5 twin children, it is expected to employ a model that can take the presence of correlation and unobserved heterogeneity into account. Thus, this study employs univariate and shared gamma frailty models.

The results of the univariate gamma frailty model showed that, the variance of the random effect is estimated to be almost zero, and the results of the Hypothesis test about the significance of the variance of the random effect found to be insignificant. This indicates that, there is no significant unobserved heterogeneity in the study population (at individual level). As a result, the estimates of covariate effect are almost similar to the estimates of the usual Cox PH model. Absence of heterogeneity at individual level does not indicate absence of correlation within groups. The estimate of the variance of the frailty from univariate data may has nothing to do with association. Univariate frailty variance is interpreted as a measure of unobserved heterogeneity in the study population (Wienke, 2011). The estimates of the regression parameters are adjusted for the unobserved heterogeneity in the population and only conditional given the same frailty.

As showed in Table 4, the variance of the random effect estimated from the shared gamma frailty model is significant at 5\% level of significance. This indicates that, one cannot ignore the correlation between twins. Comparison of the results of Cox PH model and shared gamma frailty model showed there are differences in the estimates of covariate effects on magnitude and significances. Almost all coefficient's magnitudes are higher in shared gamma frailty model. Age of mother at birth was significant in Cox PH model but not significant in shared gamma frailty model. These kind of differences was also observed in a study by Flinn and Heckman (1982)

This study showed that the risk of dying for a male twin child is higher than a female twin child. In agreement with this result, several studies on child mortality showed that the risk of dying for a male child is higher than a female child (Bereka, Habtewold, \& Nebi, 2017; Samuel, 2011; Tibebeu, 2011) .

This study result revealed that the effect of being first born children of twin birth is insignificant. Similar to this result, a study in Burkina Faso showed that the variable is statistically insignificant (Becher, Muller, Jahn, Gbangou, Kynast-Wolf, \& Kouyate, 2004). However, a child mortality study showed that first born children experience low survival compared to those children who have higher birth order (Balk, Tom, Adam, Fern, \& Melissa, 2003). Although to the best of the researchers knowledge not seen before, this study showed that that the risk of dying for a first born twin child is less likely than a second born twin child.

Unlike a study that showed children residing in urban areas have a better chance of survival than those residing in rural areas (Balk, Tom, Adam, Fern, \& Melissa, 2003), this study found that the variable residence is not significant. In addition, disagreement with studies that showed children born from women at youngest and oldest age are subject to high risk of death (Guo \& Rodriguez, 1992; Sastry, 1997), this study found the variable is not significantly associated with survival of under-5 twins.

The current study also showed that children of twin birth who were born before the older sibling reaches the age of 18 months experience low survival compared to those children of twin birth who have more than 24 month spacing. Several studies that are conducted on determinants of child mortality found similar results (Becher, Muller, Jahn, Gbangou, Kynast-Wolf, \& Kouyate, 2004; Koissi \& Hgns, 2001; Guo \& Rodriguez, 
1992).

The study also showed that the risk of dying for a child of twin birth who has a younger sibling that born within 18 months is higher than a child of twin birth who has a younger sibling that born after 24 months. Several studies that are conducted on determinants of child mortality found similar results (Becher, Muller, Jahn, Gbangou, Kynast-Wolf, \& Kouyate, 2004; Koissi \& Hgns, 2001; Guo \& Rodriguez, 1992).

\section{Conclusions and recommendations}

\subsection{Conclusion}

The results of this study showed that the main factors associated with twin under-five mortality are sex of child, between twin birth order, preceding birth interval, and succeeding birth interval.

Male twins have lower chance of survival compared to female twin children.

The risk of dying for a first born twin child is less likely than a second born twin child.

Those twins who were born before their sibling reaches 18 month experience low survival compare to those twins who have more than 24 month spacing. And those twins who had a younger sibling with age less than 18 months experience low survival compare to those twins who have a younger sibling with age more than 24 months.

Since the parameter of the heterogeneity variable is significant, one can conclude that there is a random effect shared by twins in a pair and one cannot ignore the correlation between the pair of twins.

\subsection{Recommendations}

In order to reduce the rate of child mortality among twins, this study recommends the following:

The significant effect of the birth spacing of the previous and succeeding sibling on the survival chance of children of twin birth indicate that efforts have to be exerted to educate the public about family planning and birth spacing.

The significant effect of sex of child and between twin birth order may indicate that it is important to conduct further studies perhaps biological studies; in order to explain why and how these variables are significant.

The modeling practice in this study revealed the significance of including a random effect in the model. Hence, researches in this area should incorporate and assess such random effects.

Funding: The authors received no direct funding for this research.

\section{References}

Alam, N., Van Ginneken, J. K., \& Bosch, A. M. (2007). Infant mortality among twins and triplets in rural Bangladesh in 1975-2002. Trop Med int Health , 12(12), 1506-1514.

Balk, D., Tom, P., Adam, S., Fern, G., \& Melissa, N. (2003). Spatial Analysis of Childhood Mortality in West Africa. USA: ORC Macro and Center for International Earth Science Information Network (CIESIN), Columbia University, Maryland.

Becher, H., Muller, O., Jahn, A., Gbangou, A., Kynast-Wolf, G., \& Kouyate, B. (2004). Risk factors of infant and child mortality in rural Burkina Faso. Bulletin of the World Health Organization.

Bereka, S. G., Habtewold, F. G., \& Nebi, T. D. (2017). Under-Five Mortality of Children and its Determinants in Ethiopian Somali Regional State, Eastern Ethiopia. Health Sci J, 11:3.

Childs, B., Moxon, R., \& Winkelstein, J. (1992). Genetics and Infectious Diseases. Oxford University Press .

CSA. (2012). Ethiopian demographic and health survey 2011. Ethiopian central statistical agency and ORC macro.

Flinn, C. J., \& Heckman, J. J. (1982). New methods for analyzing individual event histories. Sociological Methdology, 99-140.

Guo, G., \& Grummer-Strawn, L. (1993). Child Mortality among Twins in less developed countries. journal of Demography, 47, 495-510.

Guo, G., \& Rodriguez, G. (1992). . Estimating a Multivariate Proportional Hazards Model for Clustered Data Using the EM Algorithm, with an Application to Child Survival in Guatemala. Journal of American Statistical Association , 87, 969-976.

Iachine, I. A. (1995). Correlated frailty concept in the analysis of bivariate survival data. Bachelor project, Odense University, Department of Mathematics and Computer Science, Denmark.

Justesen, A., \& Kunst, A. (2000). Postneonatal and child mortality among twins in Southern and Eastern Africa. International journal of Epidemiology, 29, 678-683.

Koissi, M. C., \& Hgns, G. (2001). Using WinBUGS to Study Family Frailty in Child Mortality, with an Application to Child Survival in Ivory Coast. African population studies , 20.

Munyamahoro, F. (2017). An Empirical Analysis of Death of Children Under Five Years in Rwanda. J Med Res Health Educ, 1 . 
Olalekan, A. U., Mubashir, B. U., \& Ismail, Y. (2000). A Population- based study of effect of multiple birth on infant mortality in Nigeria. BMC Pregnancy and Child birth , 41-47.

Pison, G. (1992). Twins in sub-Saharan Africa: frequency, social status and mortality. Clarendon Press , 253278.

Samuel, M. (2011). Determination of factors associated with high risk of infant mortality in Ethiopia. M.Sc. Thesis, Addis Ababa University, Addis Ababa.

Sastry, N. (1997). A nest frailty model for survival data with an ap- plication to the study of child survival in Northeast Brazil. Journal of the American Statistical Association, 92, 426-35.

Smits, J., \& Monden, C. (2011). Twinning across the developing world. PLos ONE , 6(9).

Tibebeu, G. (2011). Identification of risk factors and regional differentials in under five mortality in thiopia using multilevel count model. M.Sc. Thesis, Addis Ababa University, Addis Ababa .

UNICEF. (2011). Child mortality progress report .

WHO. (2006). Neonatal and prenatal mortality country . regional and global estimates .

WHO. (2016). Under five mortality rates. Global Health observatory.

Wienke, A. (2011). Frailty Models in Survival Analysis. Chapman \& Hall/CRC,Taylor \& Francis Group.

You, D., New, J. R., \& Wardlaw, T. (2014). Report on levels and trend in child mortality . the united nation inter-agency group of child mortality estimation . 